\title{
A Taxonomy of Distribution for Cooperative Mobile Manipulators
}

\author{
Andreas Schierl, Andreas Angerer, Alwin Hoffmann, Michael Vistein and Wolfgang Reif \\ Institute for Software and Systems Engineering, University of Augsburg, Augsburg, Germany
}

Keywords: Mobile Robots, Cooperative Manipulators, Software Distribution, Robot Architectures.

\begin{abstract}
Simple robot applications can be run on a single computer, but when it comes to more complex applications or multiple mobile robots, software distribution becomes important. When structuring mobile robot systems and applications, distribution has to be considered on various levels. This paper proposes to distinguish between real-time level, system level, application level and regarding the world model. Advantages and disadvantages of distribution on each level are analyzed, and examples are given how this distribution is realized in the robotics frameworks OROCOS, ROS and the Robotics API. The results are demonstrated using a case study of two cooperating youBots handing over a work-piece while in motion, which is shown in simulation as well as in real life.
\end{abstract}

\section{INTRODUCTION}

With service robotics getting more and more important, robot demand has extended from stationary robots in factory automation towards mobile robot systems. Thus, the topic of mobile robotics has become important, and a lot of research has been performed. However, in some cases a single mobile robot is not sufficient to execute a task or deal with all problems. Once multiple robots are to work together, cooperative mobile manipulators become important, which poses new challenges.

Already in the 1990s, Dudek et al. (1996) and Cao et al. (1997) independently described a classification for cooperative mobile robotics. Dudek et al. (1996) defined a taxonomy for multi-agent mobile robotics, where such a system can be e.g. differentiated by the number of agents, the communication range, topology and bandwidth as well as the reconfigurability and the composition of homogeneous or heterogeneous agents. Similarly, Cao et al. (1997) defined research axes in cooperative mobile robotics such as the differentiation, the communication structure or different types of modeling other agents. Moreover, they made a distinction between centralized and decentralized control in the software for mobile robots. Later, Farinelli et al. (2004) added a classification based on coordination, where they compared cooperation, knowledge, coordination and organization of agents in multi-agent systems. This classification defines aware multi-robot systems where each robot knows that it is cooperating with other robots. Concentrating on aware systems, this paper analyzes which distributed software architecture can be used.

However, there is not only one distributed software architecture that is possible in cooperative mobile robotics. From our point of view, there are different levels and aspects in the software architecture of a multi-robot system that can be distributed, ranging from the application level to low-level real-time device control. Typical early software architectures such as 3T (cf. Bonasso et al., 1995) include reactive behavior as well as planning and execution, but are limited to single robot systems. The decision to distribute the software solution on one of the mentioned levels may affect the other levels and the complexity of the solution. Every possible solution, i. e. the chosen distributed software architecture, has its advantages but also its shortcomings that must be considered. In robotics systems, component based software frameworks are commonly used (cf. Brugali and Shakhimardanov, 2010), for example ROS which facilitate transparent distribution (cf. Quigley et al., 2009). However, although communication between distributed components is easy using these frameworks, the decision about distribution as well as the assignment of responsibilities to certain components affects the overall capabilities of the solution (e.g. support for hard real-time).

In this paper, we introduce a taxonomy for distributed software architectures in cooperative mobile robotics. We are interested in finding a generalized 
representation and description of distributed robotics systems. Hence, this taxonomy can be used to classify and compare the software architectures of distributed robotics systems. Additionally, we give advantages and disadvantages of applying distribution on different levels. It is important to be able to compare distributed robotics systems as the chosen software architecture often influences or sometimes even determines the complexity of the solution. However, over-simplification can sometimes lead to unrealistic solutions that will fail in real life.

Section 2 describes a case study using cooperating KUKA youBots as mobile manipulators, which is used as reference example throughout the paper. A taxonomy that separates different levels for structuring software for (mobile) robots is given in Section 3. Subsequently, the different possibilities of distributing software using these levels, as well as the advantages and disadvantages are discussed in Section 4. To show the general validity of the taxonomy, the distribution possibilities are explained using three different software frameworks. Experimental results about different solutions of the case study are presented in Section 5. Section 6 concludes this paper and gives an outlook.

\section{CASE STUDY}

As a case study for cooperating mobile robots, a scenario containing two KUKA youBots (cf. Bischoff et al., 2011) is used. In this scenario, the robots physically interact with each other to transfer a work piece from one youBot to the other.

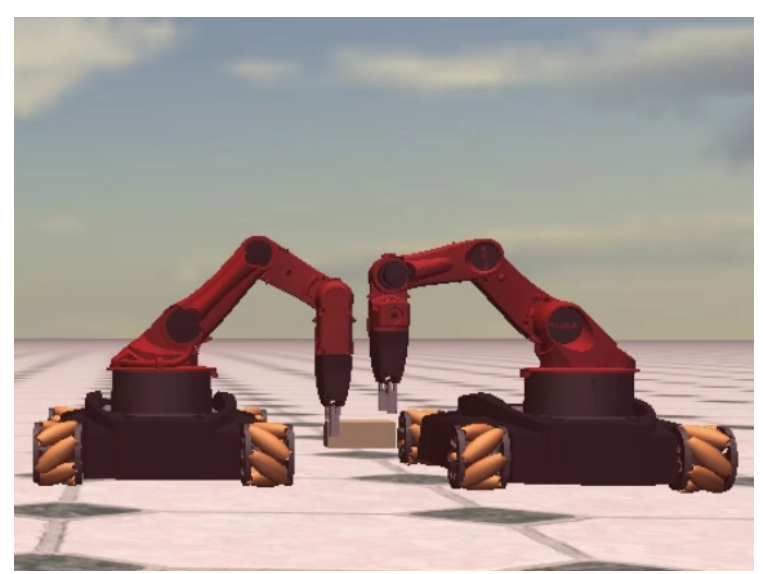

Figure 1: Cooperation of two youBots in simulation.

This scenario is inspected in different levels of difficulty. The first variant runs in simulation (cf. Figure 1), where both robots can be coordinated in realtime, exact position information is available and all control inputs and trajectories are exactly followed. For this variant, first an easy case is analyzed where the first robot is standing still, and afterwards a more complex case follows where the workpiece transfer happens while both robots are moving.

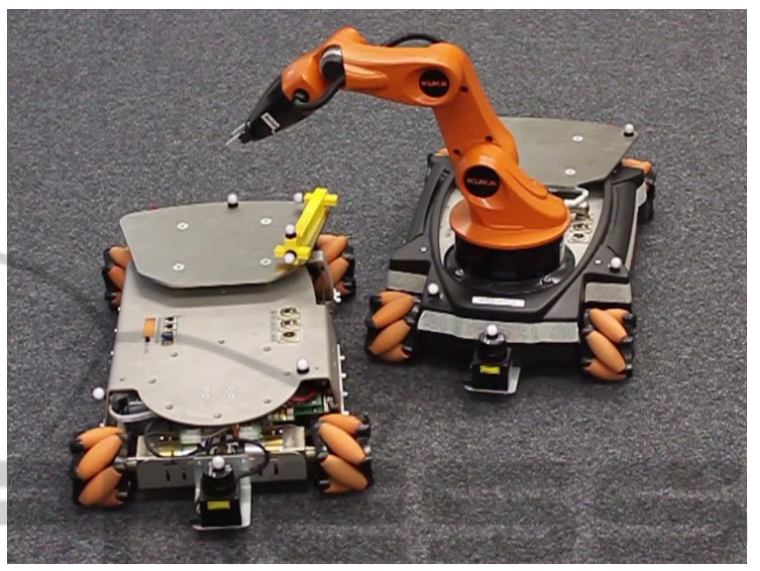

Figure 2: Cooperation of two real youBots.

As some of the assumptions made for simulation case are not valid for real robots, a second variant with real youBots is analyzed (cf. Figure 2). There, a youBot platform (left, without arm) is initially carrying a workpiece, which is then picked up by the second youBot (right) while both youBots are moving. The youBots and the workpiece are tracked externally using a Vicon optical tracking system, so precise position information is available.

However, the the concepts presented in the following sections are not specific to this use case with mobile robots, but also apply to other cooperating robots such as teams of industrial robots that produce goods in an automation plant.

\section{SOFTWARE STRUCTURE FOR DISTRIBUTED ROBOTS}

When designing a software architecture for a distributed robot scenario, we propose to group the software components into different layers as illustrated in Figure 3.

Each of the hardware devices present in the robot solution is represented and controlled by a device driver: The component that communicates with the hardware device through the vendor-specific interface. The device driver is responsible for exchanging data with the surrounding software components. It has to derive control inputs and forward them to the device, as well as receive feedback from the device and make it available to other software components. 


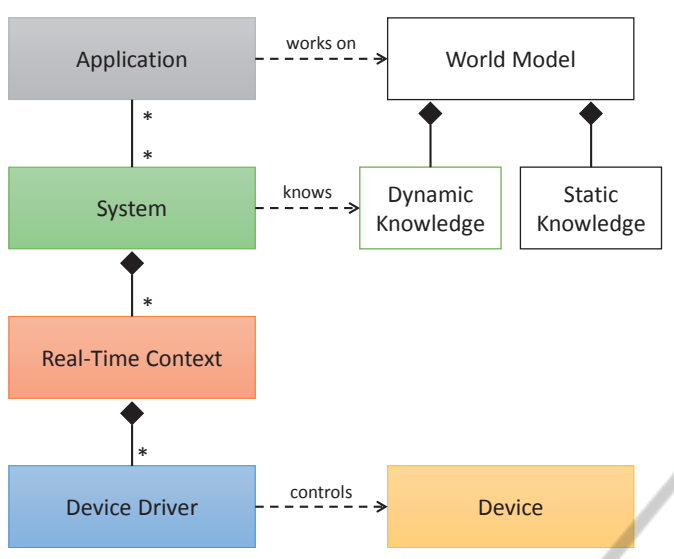

Figure 3: Software structure for distributed robots.

Each device driver can belong to a real-time context: The components between which data transfer and coordination occurs with given timing guarantees. Depending on the implementation, the real-time context can contain only one device, or span over multiple devices. Within a real-time context, reactions to events or the processing of sensor data can be guaranteed to happen before a given time limit (hard real-time). This allows to handle safety-critical situations that require timing guarantees (e.g. to stop the robot if an obstacle occurs), or to execute precise behaviors (such as actions that happen at a given point on a trajectory).

Above the real-time level, one or multiple realtime contexts belong to a system: The components between which all knowledge is shared. Within one system, all components are allowed to access each other's data, and to communicate with and send commands to each other. This allows components to directly include other components' data into planning or computation, though no real-time guarantees are given (unless handled within one real-time context). A system can span multiple computers, as long as reliable means of communication exist to facilitate the required data transfer.

To perform a desired task, one or more systems can be used in an application: The components that coordinate a work flow executed by the systems. Within an application, data is read and commands are sent to the controlled systems, so that the corresponding devices execute the task. However, if data from one system is required for an action in another system, it is the responsibility of the application or the deployment to facilitate the required data transfer, as there is no concept of implicit shared data between systems. The overall behavior of cooperating robots results from the interplay of all applications that coordinate the robots.

Each application performs its work based on its world model: The knowledge about the controlled devices and systems, as well as the environment including other (cooperating) devices. This includes geometric information such as positions and orientations of the relevant objects, as well as physical data (such as mass and friction), shape data (such as 3D models for visualization or collision checks), semantics and ontologies. Information from the world model can be stored and used in applications, systems or real-time contexts, and can also be shared between different applications and systems. Structurally, the world model data can be differentiated into dynamic and static knowledge, with static knowledge (e.g. maps, shapes and ontologies) being valid everywhere, while dynamic knowledge (such as positions and sensor data) may be known in only one system or be different in different systems.

\section{DISTRIBUTING THE SOFTWARE}

Depending on the requirements and technical limitations of the robot solution, the size and distribution of real-time contexts, systems and applications and thus the structure of the software can vary. This section discusses different design decisions concerning this structure based on the examples of the case study described in Section 2 and using the three popular frameworks OROCOS, ROS and the Robotics API. OROCOS as a component framework mainly targets control systems with real-time guarantees (cf. Bruyninckx, 2001). The main focus of ROS is to be a component framework with transparent distribution, which over time has collected a large amount of algorithms as reusable components (cf. Quigley et al., 2009). The Robotics API focuses on high-level robot programming using modern programming languages (such as Java) while still providing real-time guarantees (cf. Angerer et al., 2013).

\subsection{Real-time Level}

First, the existing hardware devices and device drivers have to be grouped into real-time contexts. In the mobile manipulator example of Section 2 this is mainly the two youBots, where we will concentrate on two platforms and one or two arms. Generally speaking, there are four different choices for this situation (cf. Figure 4):

In the smallest case (Real-time Context A in Figure 4), the device driver software is written without real-time in mind. In this case, the real-time context 

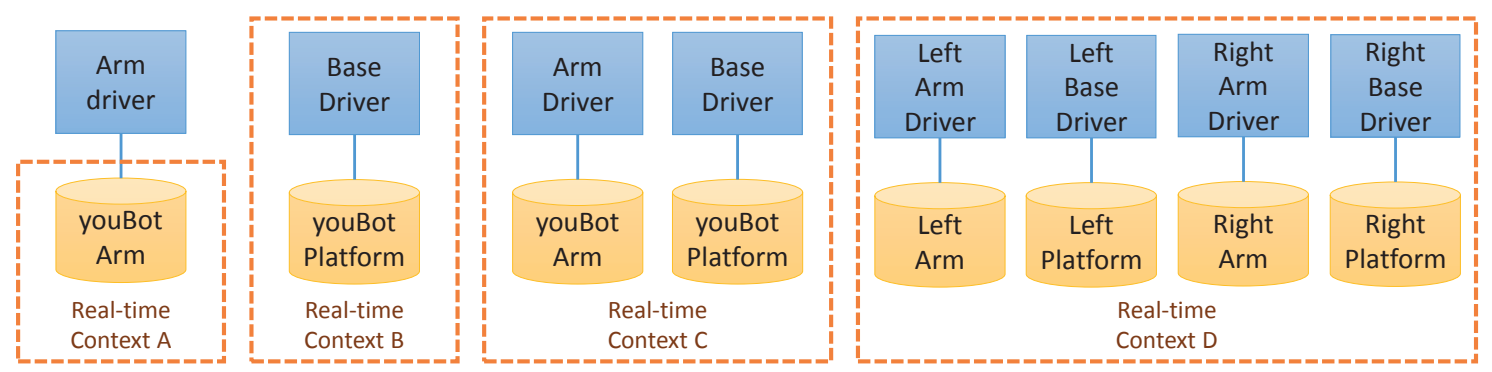

Figure 4: Different variants of real-time contexts.

only spans the firmware (if real-time capable) or controller (digital or analog) present in the device itself. For the youBot Arm, this could mean that only the position control mode of the arm motor controllers is used, where it is sufficient to give one joint configuration that the robot is expected to move to. While easy to implement, no synchronization between the joints or support for precise Cartesian space motions is possible, and no guarantees can be given regarding the interpolation quality of user-defined trajectories or the timing of reactions to events (unless supported directly by the device).

In the next case (Real-time Context B), the device driver and the communication with the device is implemented in a real-time capable fashion. This requires to use a real-time operating system and more care when implementing the device driver, but allows to execute precise custom trajectories and handle sensor events with timing guarantees. For example, a real-time capable driver running at $250 \mathrm{~Hz}$ could be implemented for the youBot Platform on a real-time operating system such as VxWorks or Xenomai. It can provide the motor controllers with smooth control set points for velocity or torque control, which allows precise user-defined trajectories, or to implement custom feed-forward or feedback control laws. However, as a drawback only information that is provided by the device itself can be included in the control law. For example only the wheel position can be controlled exactly, but the position of the entire robot in Cartesian space is inaccurate (due to wheel slip and other factors limiting odometry precision), and the platform motion cannot be synchronized with the arm motion.

Increasing the context, multiple devices can be combined (cf. Real-time Context C), up to all devices that are physically connected to the controlling computer. Both the youBot arm and the platform, which are connected to the onboard computer via EtherCAT, can be controlled from a real-time capable software on a real-time operating system. This way, coordinated motions between platform and arm are possible, that are realized through the 5 joints of the arm and the three degrees of freedom provided by the omni- directional platform. This for example allows Cartesian space motions of the end-effector relative to a point in Cartesian space known to the youBot (such as the position where the youBot started - called the odometry origin - assuming that odometry exactly provides the current position relative to this origin based on wheel rotations). Additionally, one device can react to events that occur at other devices or are detected by other sensors. However, to be able to specify these reactions, either this part of the software has to be changed for a specific application, or a flexible specification language is required (cf. Schierl et al., 2012, 2013).

To enable easy cooperation between multiple robots, the devices of all robots could be combined into one real-time context (cf. Real-time Context D). However, if the corresponding devices are connected to different PCs, real-time communication between the PCs is required. For stationary robots such as robot arms or automation systems in a factory, or complex mobile robot systems with multiple computers on board (such as the WillowGarage PR2 or the DLR Justin), this is possible through Ethernet or field buses like Ethercat. In the automation domain, standard equipment such as PLCs are used, while in robot research software frameworks such as aRDx (cf. Hammer and Bauml, 2013) or Orocos (cf. Bruyninckx, 2001) are preferred. But between mobile robots, using a wired connection usually is no option, and standards for general purpose real-time capable wireless connections are not yet common, so providing a single real-time context is not yet widely usable. In summary, while distributing a real-time context over multiple computers can improve the scalability of the solution (w.r.t. processing power or device connectivity), the need for deterministic communication implies special requirements (such as field bus hardware or dedicated networks) that make the solution more complex or expensive.

Depending on their focus, different robot frameworks offer more or less support for large real-time contexts:

- In ROS, real-time contexts with one device (vari- 


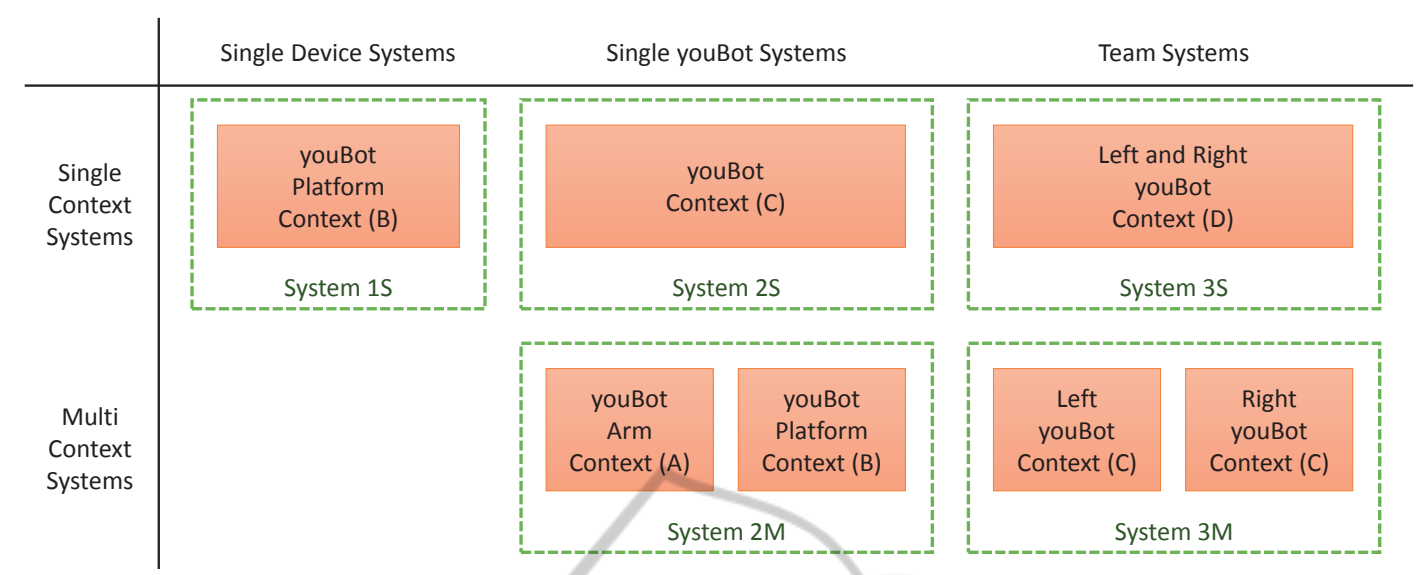

Figure 5: Different variants of systems.

ants A and B) are widely used, when a single device is encapsulated into a ros node, providing an interface to execute the required local commands. Sometimes multiple devices (such as a youBot arm and platform) are combined into one node, however this leads to higher coupling.

- In OROCOS usually most devices are combined into one real-time context, because the OROCOS frameworks targets real-time capable component systems with device drivers implemented in C++ on a real-time operating systems such as RTAI or Xenomai. When using multiple mobile robots, sometimes a wired connection (cf. Klotzbücher et al., 2013) is used to ensure one real-time context. In other cases, other communication frameworks such as ROS provide non-real-time communication between the distributed robots, thus introducing multiple real-time contexts.

- Using the Robotics API, the Robot Control Core (cf. Vistein et al., 2010) is implemented in C++ for Xenomai and includes real-time capable drivers for devices connected to the corresponding computer. This way, all devices physically connected to the computer can form a real-time context. Furthermore, plans exist to extend the implementation to support multiple Robot Control Cores as a single real-time context, given a deterministic (wired) connection exists.

\subsection{System Level}

Proceeding to the system level, real-time contexts can be grouped into systems. When looking at the number of real-time contexts involved, two options exist on the system level (cf. Figure 5):

As a minimal case, each real-time context has its own system (cf. System 1S, $2 \mathrm{~S}$ and $2 \mathrm{~S}$ in the Figure). But it is also possible to combine multiple or even all real-time contexts into a single system (cf. System $2 \mathrm{M}$ and $3 \mathrm{M}$ ). Another grouping option is based on the set of devices combined into the system. This can be just one device (e.g. one platform in System 1S), all devices of one youBot (cf. System $2 \mathrm{~S}$ and $2 \mathrm{M}$ ) or all youBots (cf. System $3 \mathrm{~S}$ and $3 \mathrm{M}$ ).

Using a big system spanning all robots has the advantage of simplifying application programming or deployment: All the data that any component might need is made available everywhere in the system, so no manual data transfer is required. This especially covers the world model - within one system, a consistent world model is possible, because the best knowledge about the world is available to every component.

However, there can be various reasons to use multiple systems: The sheer amount of data present in a big (multi) robot system can be a technical reason. Scalability can be limited by the management overhead induced by the data transfer between a great amount of components, and the addressing or mapping of data to components can become problematic. Furthermore, network bandwidth or reliability can be a limiting factor. In particular, this can be a problem when multiple robots are used that cooperate in varying teams. While for constant teams the corresponding robots could be joined into one system, varying teams quickly increase the required system size as all robots that might work together in a team at any time have to be within the same system.

But also more political reasons can opt for the separation into multiple systems, if cooperating robots belong to different people or parties. In this situation, not everyone might want to provide access to all of the robot's data, or allow everyone else to control the robot. Then, matters of trust or access control become important that do not fit into the shareeverything theme of a system. However, these reasons do not occur between the different devices within one 


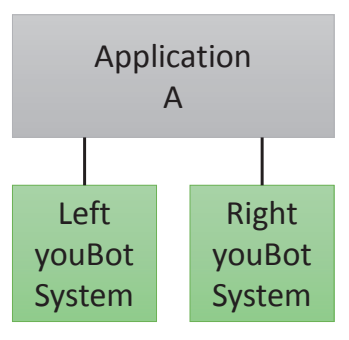

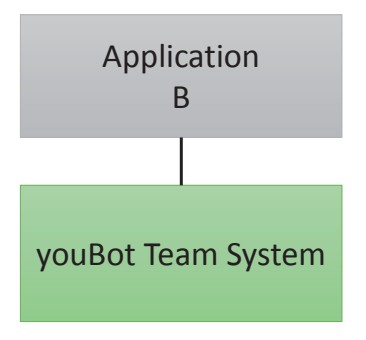

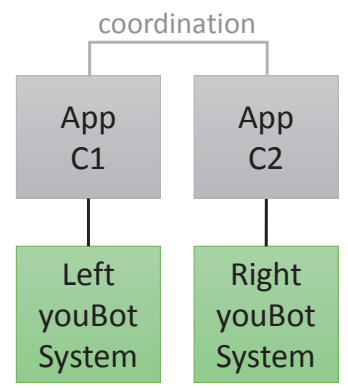

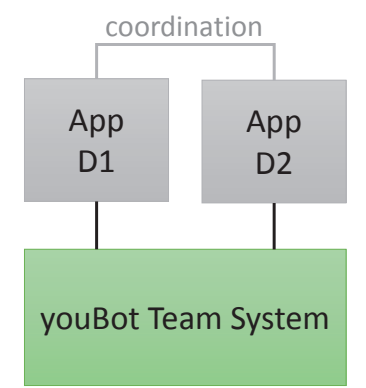

Figure 6: Different variants of applications.

robot (such as the youBot), so the grouping option $1 \mathrm{~S}$ with just a platform only makes sense if the platform is used without an arm.

Looking at the software frameworks, the different focus becomes obvious:

- In ROS, one system includes all nodes that run using the same ROS master. In this situation, all these nodes can subscribe to any data published by other nodes, and post messages or actions. The world model includes transformation data as provided by the if service, as well as robot models and data published by other nodes.

- OROCOS as a framework concentrates on one real-time context and does not per se contain features for non-real-time communication, however often ROS is used to combine multiple real-time contexts into one common system that allows for non-real-time communication and data sharing. World models are usually implemented as components in an application dependent manner, which can include geometry, semantics and history (cf. Blumenthal et al., 2013).

- In the Robotics API, the system term refers to the concept of a RoboticsRuntime, which represents one real-time context and makes the data available - to applications in a non-real-time way as well as to other devices in the same context for real-time reactions. The frame graph (cf. Angerer et al., 2013) including semantic information (such as the type of relation between frames, if they are static, temporary or have a transformation that can change during execution) and information about physical objects (such as physics meta data and shape) serves as a world model that can be used for planning as well as task execution.

Generally speaking, it is possible to distribute the corresponding components that form one system onto different PC systems, as no realtime guarantees are required on this level. Therefore, standard communication methods including wireless ones such as WiFi are applicable, however reliability or bandwidth can be limiting factors.

\subsection{Application Level}

To perform a requested task, one or multiple systems have to execute actions that are controlled and coordinated by one or multiple applications. Figure 6 gives the different possibilities to structure the application(s) for controlling the two youBots.

One way is to control all robots from one application (cf. Application A and B). This defines all the interaction present in the solution in one place and thus makes it easier to understand. However, if varying teams are needed in a certain scenario, the corresponding application has to coordinate all robots at the same time. This can become confusing if the concurrent execution of multiple subtasks is encoded in one control flow or sequential state machine. Thus, separating concerns into subtasks, one for each team, should be considered within the application.

Another way is to use multiple applications, e.g. one for each controlled system (cf. App C1, C2, D1 and D2). This way, changing teams can be implemented by only locally and independently describing the behavior of each system. However, the applications have to coordinate, either through explicit communication or through observation of the environment or other robots. Using separate applications can also be required for political reasons, as described in the previous section. In a multi-application cooperation scheme, however, the resulting behaviour is not easily understandable by looking at one place, but only by examining the interaction of the different applications involved. In the most extreme case, the application for each robot only implements low-level behaviours, and the resulting behaviour emerges from the interaction (cf. Mataric, 1993).

Another structuring approach looks at the relation between applications and systems. It is possible to control (different devices of) one system from multiple applications (cf. D1 and D2), one system from one application (cf. B, C1 and C2) or multiple systems from one application (cf. A). The software framework should allow an application that was intended for use with multiple systems (e.g. A) to also work when all 
corresponding devices exist in one system, while the distribution aspect on the system and real-time level is handled by deployment (e.g. through configuration). This may however not be possible the other way round, if the application relied on having one system (or even real-time context).

There are various ways to specify applications: Mainly sequential workflows can well be expressed as a programming language control flow. For reactive behaviour, model-based approaches such as state charts or Petri nets can be more appropriate. These ways of specification are available in different frameworks, but the support for controlling multiple systems from one application varies:

- In ROS, Python scripts can be used for sequential workflows, while SMACH state machines introduced by Bohren and Cousins (2010) allow reactive behaviour. However, communication with multiple ROS masters is not natively supported. To share data between different systems (ros masters), concepts like multimaster or foreign_relay (cf. Schneider, 2012) can be used that forward topics between multiple masters and can be used through deployment. Additionally, supporting multiple masters is one of the new use cases motivating ROS $2^{1}$.

- With OROCOS, control flow can be expressed in LUA scripts, while complex coordination is possible using coordination components written in rFSM as suggested by Klotzbucher and Bruyninckx (2012). Concentrating on one real-time context, OROCOS also does not provide direct support for access to multiple systems.

- Using the Robotics API, control flow can be expressed directly in Java applications, as well as through state charts using Java state chart libraries such as SCXML. It is easily possible to use multiple systems in one application (as used by Hoffmann et al. (2014) in the Factory 2020 example), and to share limited amount of data between different systems or applications, either through common data sources (such as a Vicon system connected to both youBots through WiFi), or through explicit direct transfer.

\subsection{World Model}

If a robot is expected to cooperate with another robot, it needs the relevant data about that other robot. In the easiest case, both robots belong to the same system, thus the required information is already accessible. We then call the other robot a controlled robot.

\footnotetext{
${ }^{1}$ http://design.ros2.org/articles/why_ros2.html
}

Once robots from multiple systems are to be coordinated from one application, the world model becomes more complex. The information about robots in other systems can originate from communication or observation. Either the other system provides (maybe read-only) access to the information (then we call the other robot a remote robot), or the information is only available through observation (thus we have an $o b$ served robot). The world model thus has to keep (a descending amount of) information about controlled robots, remote robots and observed robots.

In the case of observed and remote robots, some information (e.g. position of the first robot) is available in different systems with varying precision, and has to be organized. Therefore, different world model structures can be used:

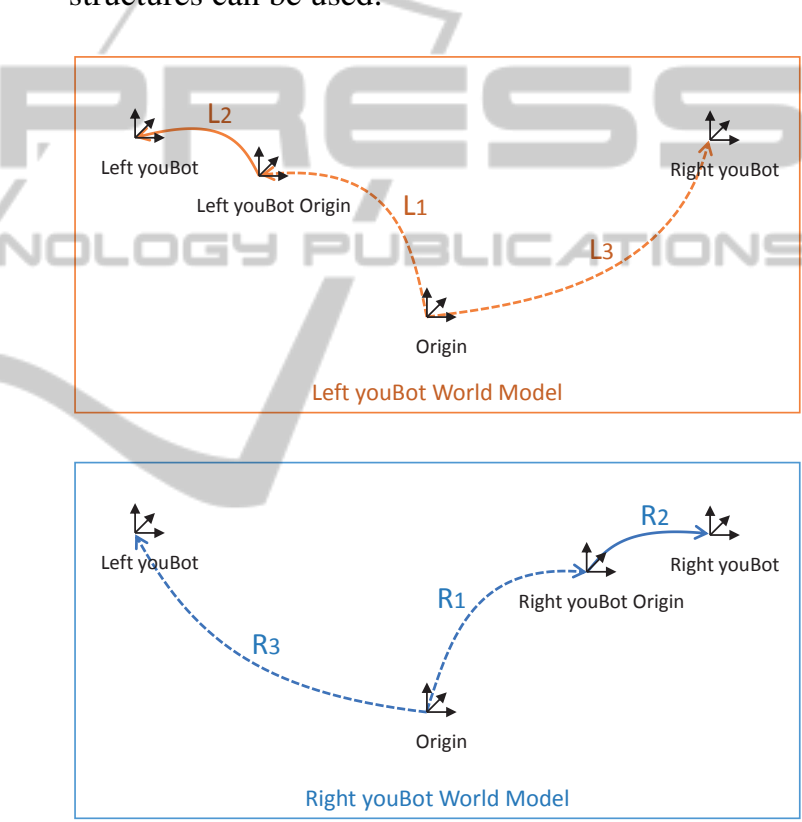

Figure 7: Separate world models for multiple systems

One way is to keep one world model per system. Figure 7 shows an example of two world models that can occur using two youBots in two systems (e.g. with Application A). The figure concentrates on geometric information, with coordinate systems indicating named positions or objects in space (frames), while arrows represent transformations between these frames. Solid arrows indicate controllable positions, while dashed arrows indicate positions retrieved through measurement or communication. Orange arrows (L1 to L3) belong to the system of the left youBot, while blue arrows (R1 to R3) belong to the right youBot system. The frames could be augmented by additional information such as shape data, which is omitted here for clarity.

Using separate models has the advantage that mul- 
tiple systems can just use different instances of the same world model, which allows to re-use models designed for single systems to a large extent. However, for cooperation a lot of information has to be duplicated, such as models of robots that occur as controlled robot in one and as a remote robot in another system. Additionally, the different world models have to be kept consistent. For example, a workpiece that is grasped and moved in one system also has to appear as grasped and moved in the other system.

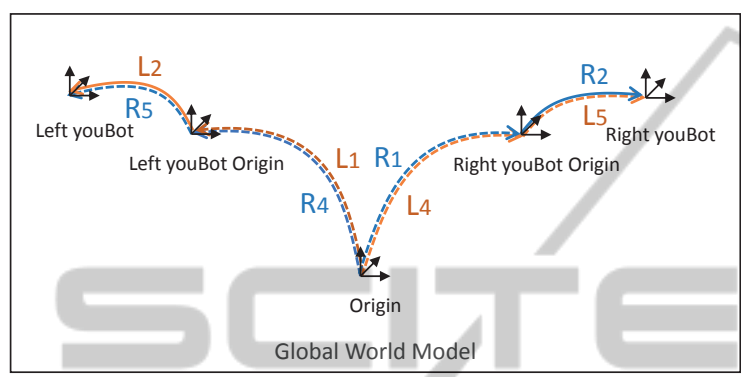

Figure 8: Using a global world model for all systems.

The second way is to keep one global world model with all the objects and relations, and to provide access to the different transformations or sensor values for each system (cf. Figure 8). This has the advantage that the world model is always consistent (as far as topology and static information is concerned, however different systems can still disagree about frame transformations), and structural changes performed in one system are automatically present in other systems.

However, this scheme lacks flexibility when dealing with observed robots: While a mobile robot can keep track of its movement since the start through odometry measurements, an observer has no way to achieve this information from outside. Thus, the frame graph contains two transformations about the robot for controlled and remote robots (R1 and R2 in Figure 7), while for observed robots only one transformation is available (R3 in Figure 7).

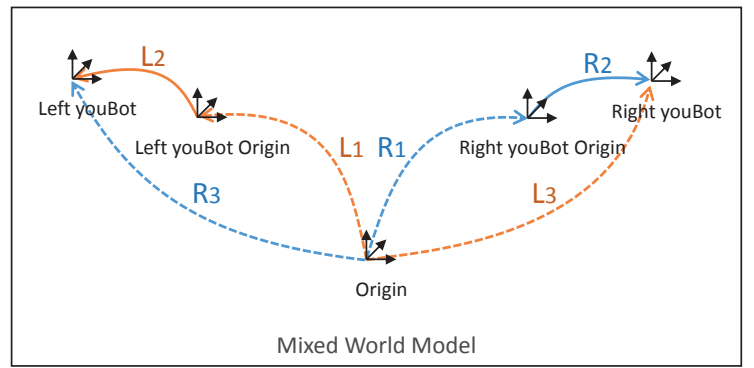

Figure 9: Using a mixed world model scheme.

To solve this problem, we propose a mixed world model scheme (cf. Figure 9). In a mixed world model, the static data is shared between all systems, while dynamic data can be different for each system. For example, information about physical objects (such as the youBot geometry) as well as static connections (such as the position where the youBot Arm is mounted relative to the youBot Platform) are shared. Dynamic connections (such as the position of the youBot relative to its origin, or the fact that the youBot is positioned relative to the World Origin or youBot Origin) can be different for each system. Still, in both systems it should be possible to compute the transformation of the Left youBot to the Right youBot, using the data and topology present in each system (and to use it for planning and execution). This combines the advantages of a shared world model with the flexibility to include limited observations, while still allowing the application to address one youBot in a uniform way.

\section{EXPERIMENTAL RESULTS} AND DISCUSSION $A T I \square N G$

The simulation experiments described in the case study have been performed using the Robotics API as a framework, using the corresponding simulation and visualization engine (cf. Figure 10). A single application (cf. Application B in Figure 6) was used. This application controlled a single system (cf. System 3S in Figure 5) containing a single real-time context (cf. Real-time Context D in Figure 4). In this real-time context, both youBot arms and platforms were simulated, as well as the youBot grippers. The application was programmed in an object-oriented fashion, referring to the robots and work pieces as software objects using a single world model and expressing the interaction in the control flow of a Java method. The initial version of the application where the first robot was not moving during transfer was easily extended into the second version where both platforms were moving. This extension mainly consisted of adding commands to move the first platform, and to make the second platform synchronize its motion to the position and movement of the first platform. This showed the simplicity of object-oriented programming and synchronizing robots in the idealized simulation case where every device belongs to the same real-time context and can be synchronized, and where exact positioning is available.

Transferring the results from simulation to reality, various changes had to be done. The tests were conducted on the two KUKA youBots available in our lab, using a Vicon optical tracking system for external localization. 


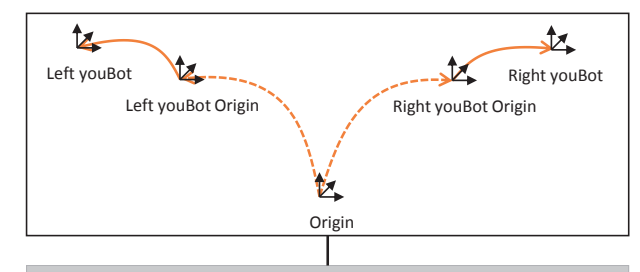

Simulation Application

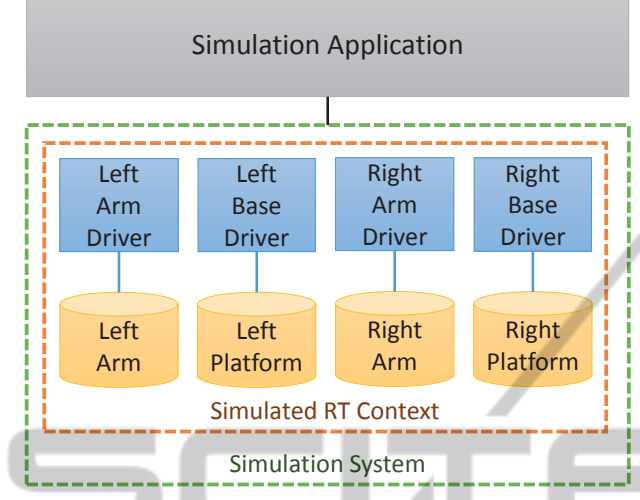

Figure 10: Structure of the simulation application.

A straightforward approach is to combine both youBots into one real-time context. Thus, the same distribution scheme like in the simulation case could be re-used, as could be large parts of the implementation. However, lacking real-time communication over wireless networks (cf. Section 4.1), this was not easily possible. On the other hand, while for stationary industrial robots adding a common real-time context spanning both robots greatly simplifies and improves the precision of physical cooperation, in the mobile case the gains are less clear. This is because precise cooperation does not only require exact timing synchronization, but also exact spatial synchronization. For stationary robots, this can be achieved by appropriate calibration procedures. For mobile systems, this is in general more problematic due to sensor inaccuracies. External positioning systems like the Vicon system used here can mitigate this problem. However, wireless real-time communication becomes a problem again when it comes to transmitting the position information to the youBots. Thus, we decided to choose an alternative distribution approach (cf. Figure 11): On each youBot's internal computer an instance of the Robot Control Core was running, which formed the real-time context (cf. Real-time Context $\mathrm{C}$ in Figure 4) and system (cf. System $2 \mathrm{~S}$ in Figure 5 for this youBot's platform, arm and gripper) for the corresponding youBot. Vicon tracking data for both youBots and the workpiece was streamed to both youBot systems through a WiFi connection from an external PC running the Vicon software.

Looking at the application level, each youBot was controlled from a separate application. The motion of
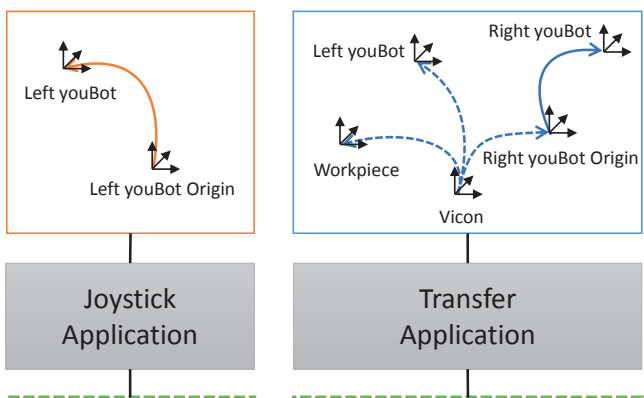

Transfer

Application

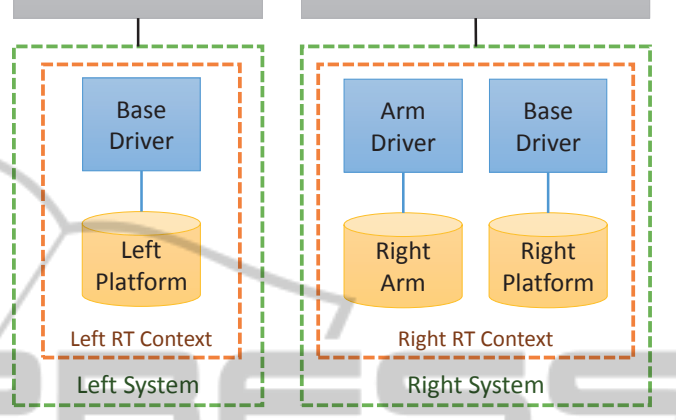

Figure 11: Structure of the real application.

the platform carrying the workpiece was controlled through teleoperation (cf. Application C1 in 6). The other youBot was controlled by a Java method similar to the one in the simulation case (cf. Application C2 in 6), however both applications used separate world models (cf. Figure 7). The workpiece and the other youBot platform were not modeled as Java objects, but only the Vicon position data was used to synchronize the motion and find the grasp position. The youBot arm used joint impedance control to mitigate position inaccuracies. Still, the experiment succeeded and the work piece could be transferred.

Instead of separate world models, a mixed world model could have been used, which would be closer to the (single) world model in the simulation case. This way, changes to the world model topology (e.g. the information that the object has been grasped) would have automatically been transferred to the second youBot's system and static position data would be known to both youBots. Depending on the trust level among both youBots, dynamic data could be exchanged by appropriate deployment using remote robots (high trust) or merely by observation, e.g. using the Vicon system in a deployment with observed robots (low trust).

\section{CONCLUSIONS AND OUTLOOK}

In this paper, we introduced four levels for structuring the software for distributed robot applications: realtime, system, application and the world model. The 
structure on the different levels can be used and combined independently, motivated by technical as well as political constraints.

The different options for structuring have been explained based on a case study of cooperating mobile manipulators and various robot frameworks, and evaluated in simulation and real world on a setup with two KUKA youBots. In the example applications, different ways to distribute the software on different levels have been shown, and the advantages and drawbacks for the given scenario have been shown. Overall, it became clear that there is not a single optimal way of structuring and distributing the software. The taxonomy presented in this work will hopefully be a starting point that can help developers in designing and discussing their software architecture. Based on non-functional requirements to the developed solution (like reactiveness, synchronization quality, data privacy, trust, ...), the choice of the appropriate distribution scheme and framework(s) for implementation should become easier.

As next steps, we plan to implement the mentioned other ways of distribution and to evaluate the gains for the given scenario. This especially includes the use of a mixed world model, as well as ways to share a world model between multiple applications or to synchronize relevant structural changes.

\section{REFERENCES}

Angerer, A., Hoffmann, A., Schierl, A., Vistein, M., and Reif, W. (2013). Robotics API: Object-Oriented Software Development for Industrial Robots. J. of Software Engineering for Robotics, 4(1):1-22.

Bischoff, R., Huggenberger, U., and Prassler, E. (2011). KUKA youBot - a mobile manipulator for research and education. In Robotics and Automation (ICRA), 2011 IEEE International Conference on, pages 1-4.

Blumenthal, S., Bruyninckx, H., Nowak, W., and Prassler, E. (2013). A scene graph based shared 3D world model for robotic applications. In Proc. 2013 IEEE Intl. Conf. on Robot. \& Autom., Karlsruhe, Germany, pages 453-460.

Bohren, J. and Cousins, S. (2010). The SMACH high-level executive. IEEE Robotics \& Automation Magazine, 17(4):18-20.

Bonasso, R. P., Kortenkamp, D., Miller, D. P., and Slack, M. (1995). Experiences with an architecture for intelligent, reactive agents. J. of Experimental and Theoretical Artificial Intelligence, 9:237-256.

Brugali, D. and Shakhimardanov, A. (2010). Componentbased robotic engineering (Part II). IEEE Robot. \& Autom. Mag., 20(1).

Bruyninckx, H. (2001). Open robot control software: the OROCOS project. In Proc. 2001 IEEE Intl. Conf. on Robot. \& Autom., pages 2523-2528, Seoul, Korea.
Cao, Y. U., Fukunaga, A. S., and Kahng, A. B. (1997). Cooperative mobile robotics: Antecedents and directions. Autonomous Robots, 4:7-27.

Dudek, G., Jenkin, M. R. M., Milios, E., and Wilkes, D. (1996). A taxonomy for multi-agent robotics. Autonomous Robots, 3:375-397.

Farinelli, A., Iocchi, L., and Nardi, D. (2004). Multirobot systems: a classification focused on coordination. Systems, Man, and Cybernetics, Part B: Cybernetics, IEEE Transactions on, 34(5):2015-2028.

Hammer, T. and Bauml, B. (2013). The highly performant and realtime deterministic communication layer of the aRDx software framework. In 16th Intl. Conf. on Advanced Robotics (ICAR), 2013, pages 1-8.

Hoffmann, A., Angerer, A., Schierl, A., Vistein, M., and Reif, W. (2014). Service-oriented robotics manufacturing by reasoning about the scene graph of a robotics cell. In ISR/Robotik 2014; 41st International Symposium on Robotics; Proceedings of, pages 1-8.

Klotzbücher, M., Biggs, G., and Bruyninckx, H. (2013) Pure coordination using the coordinator-configurator pattern. CoRR, abs/1303.0066.

Klotzbucher, M. and Bruyninckx, H. (2012). Coordinating robotic tasks and systems with rFSM statecharts. $J$. of Software Engineering for Robotics, 3, no 1:28-56.

Mataric, M. J. (1993). Designing emergent behaviors: From local interactions to collective intelligence. In Meyer, J.-A., Roitblat, H. L., and Wilson, S. W., editors, Proc. 2nd Intl. Conf. on Simulation of Adaptive Behavior, pages 432-441.

Quigley, M., Conley, K., Gerkey, B. P., Faust, J., Foote, T., Leibs, J., Wheeler, R., and Ng, A. Y. (2009). ROS: an open-source Robot Operating System. In ICRA Workshop on Open Source Software.

Schierl, A., Angerer, A., Hoffmann, A., Vistein, M., and Reif, W. (2012). Using Java for real-time critical industrial robot programming. In Wksh. on Softw. Developm. \& Integr. in Robotics. IEEE Intl. Conf. on Robot. \& Autom., St. Paul, USA.

Schierl, A., Hoffmann, A., Angerer, A., Vistein, M., and Reif, W. (2013). Towards realtime robot reactions: Patterns for modular device driver interfaces. In Wksh. on Softw. Developm. \& Integr. in Robotics. IEEE Intl. Conf. on Robot. \& Autom., Karlsruhe, Germany.

Schneider, T. (2012). Distributed Networks Using ROS - Cross-Network Middleware Communication Using IPv6. Master's thesis, Lehrstuhl für Medientechnik, Technische Universität München.

Vistein, M., Angerer, A., Hoffmann, A., Schierl, A., and Reif, W. (2010). Interfacing industrial robots using realtime primitives. In Proc. 2010 IEEE Intl. Conf. on Autom. and Logistics, Hong Kong, China, pages 468473. IEEE. 